\title{
James Webb Space Telescope: the road to first science observations (Presentation Video)
}

\section{Mark Clampin}

Mark Clampin, "James Webb Space Telescope: the road to first science observations (Presentation Video)," Proc. SPIE 9143, Space Telescopes and Instrumentation 2014: Optical, Infrared, and Millimeter Wave, 91430L (22 June 2014); doi: 10.1117/12.2063455

Event: SPIE Astronomical Telescopes + Instrumentation, 2014, Montréal, Quebec, Canada 


\title{
James Webb Space Telescope: The Road to First Science Observations (Presentation Video)
}

\author{
Mark Clampin, NASA Goddard Space Flight Ctr. (United States)
}

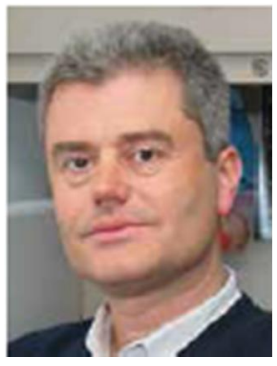

\begin{abstract}
The James Webb Space Telescope (JWST) is a large aperture, infrared telescope planned for launch in 2018. JWST is a facility observatory that will address a broad range of science goals covering four major themes: First light and ReIonization, the Assembly of Galaxies, the Birth of Stars and Protoplanetary Systems, and Planetary Systems and the Origins of Life. JWST embodies several major technical challenges. With a 6.5 meter diameter mirror it will be the largest space telescope ever flown. It is the first cryogenic telescope to incorporate passive cooling, achieved by means of a large sunshade, to reach its $\sim 40 \mathrm{~K}$ operating temperature. Due to the size of the Observatory, it must be stowed for launch, and then deployed to its operational configuration on its way to an orbit around the second Lagrange point. JWST is well on its way from drawing board to sky. Much of the flight hardware is already built and tested at the sub-system level. In this talk we will focus on the remaining tasks on the road to JWST's first science observations on the sky. Prior to the launch, the remaining work to integrate the observatory elements centers around four major activities:
\end{abstract}

- Cryo-optical testing of the instrument complement

- Cryo-optical testing of the assembled telescope and instrument complement;

- Integration of the observatory

- Full-scale testing of the observatory deployments.

We will discuss the design and philosophy underlying the cryo-optical test program for the Observatory. Cryo-optical testing begins with the instrument complement at the Goddard Space Flight Center, and finishes with an end-to-end test of the assembled telescope in the world's largest cryogenic vacuum chamber at the Johnson Space Flight Center. In the context of a detailed overview of JWST's deployment steps after launch, we will describe the final stages of Observatory integration and the testing to verify these deployments, ready for launch. Finally, we will discuss the post-launch timeline to transition the stowed Observatory to the start of science operations.

View presentation video on SPIE's Digital Library: http://dx.doi.org/10.1117/12.2063455.3664714297001 